\title{
Inhibition of permeability transition pore opening by mitochondrial STAT3 and its role in myocardial ischemia/reperfusion
}

\author{
Kerstin Boengler $\cdot$ Denise Hilfiker-Kleiner • \\ Gerd Heusch · Rainer Schulz
}

Received: 10 September 2010/Revised: 27 September 2010/Accepted: 1 October 2010/Published online: 20 October 2010

(C) Springer-Verlag 2010

\begin{abstract}
The signal transducer and activator of transcription 3 (STAT3) contributes to cardioprotection by ischemic pre- and postconditioning. Mitochondria are central elements of cardioprotective signaling, most likely by delaying mitochondrial permeability transition pore (MPTP) opening, and STAT3 has recently been identified in mitochondria. We now characterized the mitochondrial localization of STAT3 and its impact on respiration and MPTP opening. STAT3 was mainly present in the matrix of subsarcolemmal and interfibrillar cardiomyocyte mitochondria. STAT1, but not STAT5 was also detected in mitochondria under physiological conditions. ADP-stimulated respiration was reduced in mitochondria from mice with a cardiomyocyte-specific deletion of STAT3 (STAT3$\mathrm{KO})$ versus wildtypes and in rat mitochondria treated with the STAT3 inhibitor Stattic (STAT3 inhibitory compound, 6-Nitrobenzo[b]thiophene 1,1-dioxide). Mitochondria from STAT3-KO mice and Stattic-treated rat mitochondria tolerated less calcium until MPTP opening occurred. STAT3 co-immunoprecipitated with cyclophilin D, the target of the cardioprotective agent and MPTP inhibitor cyclosporine A
\end{abstract}

S. Lecour, Cape Town, South Africa, served as guest editor for the manuscript and was responsible for all editorial decisions, including the selection of reviewers. The policy applies to all manuscripts with authors from the editor's institution.

K. Boengler · G. Heusch · R. Schulz ( $₫)$

Institut für Pathophysiologie, Zentrum für Innere Medizin,

Universitätsklinikum Essen, Hufelandstr. 55,

45122 Essen, Germany

e-mail: Rainer.schulz@uk-essen.de

D. Hilfiker-Kleiner

Molekulare Kardiologie, Medizinische Hochschule Hannover,

Carl-Neuberg-Str. 1, 30625 Hannover, Germany
(CsA). However, CsA reduced infarct size to a similar extent in wildtype and STAT3-KO mice in vivo. Thus, STAT3 possibly contributes to cardioprotection by stimulation of respiration and inhibition of MPTP opening.

Keywords Cardioprotection - Ischemia . Mitochondrial permeability transition pore . Mitochondrion - Reperfusion · STAT

\section{Introduction}

Signal transducer and activator of transcription (STAT) proteins transduce stress signals from the plasma membrane to the nucleus, thereby acting as signaling molecules and transcriptional regulators [4]. Several STAT proteins are expressed in the heart, among them are STAT1, 3, and 5. Even though STAT proteins are highly homologous, their functions differ substantially, notably in ischemia/ reperfusion: STAT1 enhances whereas STAT3 and STAT5 reduce cardiomyocyte death $[4,23]$.

STAT3 and 5 are involved in the reduction of myocardial injury by ischemic pre- and postconditioning [10, 24, $39,43]$, i.e. endogenous cardioprotective phenomena which have been identified in all species so far, including man [17, 28, 42]. Deletion of STAT3 (STAT3-KO) or STAT5 abolishes the infarct size reduction by ischemic preconditioning in mice hearts $[41,46]$; the infarct size reduction by ischemic postconditioning is also attenuated in STAT3-KO mice [2, 22].

Since the cardioprotection by classical ischemic pre- and postconditioning is not dependent on increased mRNA levels [26, 38], it is unlikely that STATs exert their protective effect via regulation of target gene transcription [14], although increased nuclear translocation and 
DNA-binding of STAT1 and STAT3 have been observed with ischemic preconditioning [45, 46].

Mitochondria are centrally involved in the signal transduction of ischemic pre- and postconditioning [18]. Opening of the mitochondrial permeability transition pore (MPTP) is a major determinant of cardiomyocyte death and inhibition of MPTP opening is central to protection by ischemic pre- and postconditioning [8, 15, 21], most likely also in humans [32]. Recently, STAT3 was identified in mouse cardiomyocyte mitochondria, where it regulates complex I activity and oxygen consumption [44]. The present study confirms these findings and extends them to further address (1) the presence of STATs in subsarcolemmal (SSM) and interfibrillar (IFM) mitochondria [7, 29, 35]; (2) the interaction of STAT3 with mitochondrial respiration and MPTP opening; (3) the interaction of STAT3 with other proteins; and finally (4) the importance of STAT3 for cardioprotective strategies.

\section{Materials and methods}

The present study was performed with approval by the Bioethical Committee of the State of Nordrhein-Westfalen, Germany. It conforms to the Guide for the Care and Use of Laboratory Animals published by the US National Institutes of Health (NIH publication No. 85-23, revised 1996).

In the present study, female mice with a cardiomyocyterestricted deletion of STAT3 (STAT3-KO), which were generated by breeding STAT3-floxed mice (STAT3 ${ }^{\text {flox/flox }}$ ) with $\alpha$-myosin heavy chain promoter/Cre recombinase transgenic mice ( $\left.\alpha \mathrm{MHC}-\mathrm{Cre}^{\mathrm{tg} /-}\right)$ [20] and their wild type (WT) littermates, male and female young (8 weeks, 9 females, 1 male) and aged (21 months, 9 females, 2 males) mice (C57/B16 background) and 3-month-old Lewis rats were studied.

\section{Mitochondria isolation}

For respiration and calcium handling, subfractionation, immunoprecipitation, western blot analysis, and confocal laser scan microscopy, subsarcolemmal mitochondria (SSM) were isolated, as described previously [3]. In brief, left ventricular (LV) tissue was minced in isolation buffer (in mmol/L: sucrose 250; HEPES 10; EGTA 1, pH 7.4). The LVs were homogenized (Ultra Turrax, 3 steps of $\sim 5 \mathrm{~s}$ each, low speed) and then centrifuged at $600 \mathrm{~g}$ for $10 \mathrm{~min}$. The supernatant was centrifuged at $10,780 \mathrm{~g}$ for $10 \mathrm{~min}$. The resulting sediment was resuspended in isolation buffer and centrifuged at 7,650g for $10 \mathrm{~min}$. To analyze STAT1 and STAT3 content in SSM and interfibrillar mitochondria (IFM), SSM and IFM were isolated from rat ventricles, as previously described [7]. For mitochondrial subfractionation, immunoprecipitation, and western blot analysis, mitochondria were further purified by percoll-gradient ultracentrifugation. Mitochondria were layered on top of a $30 \%$ percoll solution in isolation buffer and centrifuged at $35,000 \mathrm{~g}$ for $30 \mathrm{~min}$. The lower mitochondrial band was collected and washed twice in isolation buffer by centrifugation at $8,000 \mathrm{~g}$ for $5 \mathrm{~min}$.

\section{Pharmacological inhibition of STAT3}

Rat LV SSM were incubated with $100 \mu \mathrm{mol} / \mathrm{L}$ Stattic (STAT3 inhibitory compound, 6-Nitrobenzo[b]thiophene 1,1-dioxide), a concentration previously shown to reduce STAT3 phosphorylation in isolated mouse hearts [11], or DMSO as vehicle for $1 \mathrm{~h}$ at $4^{\circ} \mathrm{C}$ and subsequently analyzed for STAT3 phosphorylation at tyrosine 705 and serine 727 by immunoprecipitation (percoll-purified mitochondria). Non-purified mitochondria were used to study mitochondrial oxygen consumption and calcium-induced MPTP opening.

\section{Mitochondrial function}

The respiration of $0.1 \mathrm{mg} / \mathrm{mL}$ SSM proteins was measured in a Clark-type oxygen electrode (Strathkelvin, Glasgow, $\mathrm{UK})$ at $25^{\circ} \mathrm{C}$ in incubation buffer using $5 \mathrm{mmol} / \mathrm{L}$ glutamate and $2.5 \mathrm{mmol} / \mathrm{L}$ malate as substrates for complex 1 or $5 \mathrm{mmol} / \mathrm{L}$ succinate (in combination with $2 \mu \mathrm{mol} / \mathrm{L}$ rotenone to inhibit complex 1) as substrate for complex 2 . After recording of basal oxygen consumption, respiration was determined after the addition of $40 \mu \mathrm{mol} / \mathrm{L}$ ADP [7]. Complex 4 respiration was determined after the addition of $1.8 \mu \mathrm{M}$ antimycin A to inhibit complex 3 and $300 \mu \mathrm{M}$ TMPD ( $N, N, N^{\prime}, N^{\prime}$-tetramethyl-p-phenylenediamine) and $3 \mathrm{mM}$ ascorbate-which donates electrons to cytochrome oxidase via the reduction of cytochrome c. Maximal respiration was determined after the subsequent addition of $1.3 \mu \mathrm{mol} / \mathrm{L}$ oligomycin to inhibit the ATP synthase and $30 \mathrm{nmol} / \mathrm{L}$ FCCP.

Calcium retention capacities of $0.1 \mathrm{mg} / \mathrm{mL}$ rat $\mathrm{SSM}$ or $0.075 \mathrm{mg} / \mathrm{mL}$ mouse SSM were measured in $2 \mathrm{ml}$ incubation buffer without EGTA at $25^{\circ} \mathrm{C}$ using $5 \mathrm{mmol} / \mathrm{L}$ glutamate and $2.5 \mathrm{mmol} / \mathrm{L}$ malate as substrates for complex 1 or $5 \mathrm{mmol} / \mathrm{L}$ succinate with $2 \mu \mathrm{mol} / \mathrm{L}$ rotenone as substrate for complex 2. Extramitochondrial calcium was detected using $0.5 \mu \mathrm{mol} / \mathrm{L}$ calcium green $5 \mathrm{~N}$ (Invitrogen, Carlsbad, CA, USA) with a Cary Eclipse spectrophotometer at excitation and emission wavelengths of 500 and $530 \mathrm{~nm}$, respectively. Experiments were performed in the presence and absence of $40 \mu \mathrm{mol} / \mathrm{L}$ ADP and $1.2 \mathrm{mmol} / \mathrm{L}$ $\mathrm{MgCl}_{2}$. Six nmol (mouse SSM without ADP and $\mathrm{MgCl}_{2}$ ) or $10 \mathrm{nmol}$ (all other groups) $\mathrm{CaCl}_{2}$ were added once per 
minute until an increase in calcium green fluorescence was detected, reflecting MPTP opening.

\section{Subfractionation of mitochondria}

Mitochondria were subfractionated following a modified protocol [36]. Freshly isolated percoll-purified rat LV SSM were resuspended in $150 \mathrm{mmol} / \mathrm{L} \mathrm{KCl}$ supplemented with $100 \mu \mathrm{M}$ digitonin and protease inhibitors (Complete, Roche, Basel, Switzerland). After incubation for $10 \mathrm{~min}$ on ice, the mitochondria were centrifuged at $10,000 \mathrm{~g}$ for $10 \mathrm{~min}$ at $4^{\circ} \mathrm{C}$. The supernatant contained the proteins of the intermembrane space. The sediment was resuspended in isolation buffer supplemented with $200 \mu \mathrm{mol} / \mathrm{L}$ digitonin. An equal volume of sunflower oil was added, and the mitochondria were vigorously mixed for $5 \mathrm{~min}$. After centrifugation for $5 \mathrm{~min}$ at $14,000 \mathrm{~g}$, the lower phase containing the matrix was separated from the interphase containing the membrane proteins.

Mitochondrial swelling was induced by incubation in hypoosmotic solution ( $5 \mathrm{mmol} / \mathrm{L}$ sucrose, $5 \mathrm{mmol} / \mathrm{L}$ Hepes, $1 \mathrm{mmol} / \mathrm{L}$ EGTA, $\mathrm{pH}$ 7.2) for $10 \mathrm{~min}$ before normalizing the osmolarity by adding hyperosmotic solution [37].

\section{Immunoprecipitation and western blot analysis}

Mitochondria or right ventricles were lysed in $1 \times$ Cell lysis buffer [Cell Signaling, Beverly, MA, USA; containing in mmol/L: Tris (pH 7.5) 20, $\mathrm{NaCl} \mathrm{150,} \mathrm{EDTA} \mathrm{1,} \mathrm{EGTA} \mathrm{1,}$ sodium pyrophosphate $2.5, \beta$-glycerolphosphate $1, \mathrm{Na}_{3} \mathrm{VO}_{4}$ 1 , Triton X-100 1\%, Leupeptin $1 \mu \mathrm{g} / \mathrm{mL}$, supplemented with $1 \times$ Complete Protease Inhibitor Cocktail (Roche, Basel, Switzerland)], and subsequently centrifuged at $13,000 \mathrm{~g}$ for $10 \mathrm{~min}$. The protein concentration of the supernatant was determined using the Dc protein assay (BioRad, Hercules, CA, USA).

For immunoprecipitation, $400 \mu \mathrm{g}$ rat mitochondrial proteins were incubated with antibodies against total or phosphorylated STAT3 (Tyr705 or Ser727), Tom20, antirabbit or anti-mouse horseradish peroxidase conjugated IgGs for $1 \mathrm{~h}$ at $4^{\circ} \mathrm{C}$. Protein A Agarose (Santa Cruz) was added to each sample followed by overnight incubation at $4{ }^{\circ} \mathrm{C}$. Protein A Agarose beads were washed three times with $500 \mu \mathrm{l}$ PBS supplemented with $1 \times$ Complete Protease Inhibitors. After adding sample buffer, the samples were boiled for $5 \mathrm{~min}$ at $95^{\circ} \mathrm{C}$.

Immunoprecipitated proteins, $5 \mu \mathrm{g}$ subfractionated mitochondrial proteins or $25 \mu \mathrm{g}$ total mitochondrial or right ventricular proteins were electrophoretically separated on $10 \%$ SDS gels and transferred to nitrocellulose membranes. Protein transfer was controlled by staining of membranes with Ponceau S. After blocking, the membranes were incubated with primary antibodies and then washed with the respective secondary antibodies. Immunoreactivities were detected using the SuperSignal West Femto Maximum Sensitivity Substrate (Pierce, Rockford, IL, USA) and densitometrically quantified with Scion Image software (Frederick, ML, USA).

\section{Ischemia/reperfusion injury in vivo}

Female STAT3-KO mice or their WT littermates were anesthetized with pentobarbital sodium ( $80 \mathrm{mg} / \mathrm{kg}$ ip). The temperature of the animals was kept stable between 36.6 and $37.4^{\circ} \mathrm{C}$ using heating pads, and the electrocardiogram was monitored continuously. After intubation (polyethylene-60 tubing), the animals were ventilated with a stroke rate of $130 / \mathrm{min}$ and a tidal volume of $1 \mathrm{~mL}$. A midline thoracotomy and pericardiotomy were performed. The left anterior descending coronary artery (LAD) was occluded 2-3 $\mathrm{mm}$ distal to the tip of the left auricle using a 7.0 silk suture and a small tube to form a snare for $30 \mathrm{~min} .5 \mathrm{~min}$ before reperfusion, $10 \mathrm{mg} / \mathrm{kg}$ cyclosporine $\mathrm{A}$ or $0.9 \% \mathrm{NaCl}$ as vehicle were administered intravenously. Hearts were then reperfused for $120 \mathrm{~min}$. $1 \mathrm{U} / \mathrm{g}$ heparin was given intraperitoneally $20 \mathrm{~min}$ before the end of reperfusion. At the end of the protocol, the LAD was re-occluded, $1 \mathrm{~mL}$ of $5 \%$ Evans Blue was injected into the left ventricle (LV), and mice were killed with a $\mathrm{KCl}$ bolus. Hearts were excised, and atrial and right ventricular tissues were removed. $\mathrm{LV}$ tissue was washed, frozen for $30 \mathrm{~min}$ at $-20^{\circ} \mathrm{C}$, cut into 5-6 transverse slices, and incubated for $20 \mathrm{~min}$ at $37^{\circ} \mathrm{C}$ in $2 \%$ TTC (2,3,5-triphenyl tetrazolium chloride) and for $12 \mathrm{~h}$ in $10 \%$ formalin. Tissue sections were photographed and infarct size was calculated as percent of the area at risk by planimetry.

\section{Antibodies}

The following antibodies were used in the present study: rabbit polyclonal anti-mouse phosphorylated STAT3 (Tyr705, Cell Signaling, Beverly, MA, USA), rabbit polyclonal anti-mouse phosphorylated STAT3 (Ser727, Cell Signaling, Beverly, MA, USA), rabbit polyclonal antimouse total STAT3 (Cell Signaling, Beverly, MA, USA), mouse monoclonal anti-mouse total STAT3 (Cell Signaling, Beverly, MA, USA), rabbit polyclonal anti-rat total connexin43 (Invitrogen, Carlsbad, CA, USA), mouse monoclonal anti-rat sodium/potassium $\left(\mathrm{Na}^{+} / \mathrm{K}^{+}\right)$-ATPase (Upstate, Waltham, MA, USA), mouse monoclonal antidog sarcoplasmic calcium (SERCA2)-ATPase (Sigma, Saint Louis, MO, USA), rabbit monoclonal anti-human histone deacetylase 2 (HDAC2, Abcam, Cambridge, UK), mouse monoclonal anti-rabbit GAPDH (Hytest, Turku, Finland), rabbit polyclonal anti-human translocase of the outer membrane 20 (Tom20, Santa Cruz, Santa Cruz, CA, 
USA), rabbit polyclonal anti-human VDAC (voltagedependent anion channel, Abcam, Cambridge, UK), mouse monoclonal anti-human ATP synthase $\alpha$ (BD Transduction, San Diego, CA, USA), mouse monoclonal anti-rat cytochrome c (Santa Cruz, Santa Cruz, CA, USA), rabbit polyclonal anti-human cyclophilin D (Acris, Hiddenhausen, Germany), rabbit polyclonal anti-human MnSOD (manganese superoxide dismutase, Upstate, Lake Placid, NY, USA), or mouse monoclonal anti-rat cyclophilin D (MitoSciences, Eugene, OR, USA).

\section{Statistics}

Data are presented as mean values \pm SEM. Western blot data of subfractionated mitochondria and infarct size data were compared by two-way repeated measures ANOVA and Fisher's LSD. Western blot data on the expression and phosphorylation level of STAT1, STAT3, and Tom20 in SSM/IFM and on STAT3 or Cx43 in young/aged mitochondria, as well as data on mitochondrial respiration were compared by unpaired Student's $t$ test. A $p<0.05$ indicated a significant difference.

\section{Results}

The mitochondrial preparations used to study the presence of STAT proteins were not contaminated, since western blot analysis on different amounts $(2-30 \mu \mathrm{g})$ of rat LV SSM proteins was negative for marker proteins of the sarcolemma $\left(\mathrm{Na}^{+} / \mathrm{K}^{+}\right.$-ATPase), sarcoplasmic reticulum (sarcoplasmic/ endoplasmic reticulum calcium ATPase 2a, Serca2a), cytosol (glycerolaldehyde-3-phosphate dehydrogenase, GAPDH), nucleus (histone deacetylase 2, HDAC2), but positive for the mitochondrial markers voltage-dependent anion channel (VDAC) and ATP synthase $\alpha$. In rat LV mitochondria, both STAT1 and STAT3 were detected, whereas STAT5 immunoreactivity was absent (Fig. 1a). The amounts of STAT1 and STAT3 were similar in both, the SSM and IFM populations (Fig. 1c, d). In mitochondria isolated from STAT3-KO mice, STAT3-specific immunoreactivity was largely reduced, whereas STAT1 immunoreactivity was preserved (Fig. 1b). The presence of STAT3 in WT mitochondria and the loss of STAT3 immunoreactivity in STAT3-KO mitochondria were confirmed by confocal laser scan microscopy (Appendix Fig. 9).

Since only STAT3 and STAT5 have an established role in endogenous cardioprotection, but only STAT3 is present in mitochondria, we subsequently focussed our investigations on mitochondrial STAT3.

Mitochondrial STAT3 was dually phosphorylated at tyrosine 705 and serine 727, as shown by immunoprecipitation and subsequent western blot analysis (Fig. 2b) as well as by confocal laser scan microscopy (Appendix Figs. 9 and 10). In STAT3-KO mitochondria, no signal for STAT3 phosphorylated at Ser727 and for STAT3 phosphorylated at Tyr705 was detected (Appendix Fig. 9).

Total STAT3 was immunoprecipitated from rat LV SSM. The specificity of the immunoprecipitation was demonstrated by the lack of STAT3 immunoreactivity in the control $\operatorname{IgG}$ precipitation and lack of manganese superoxide dismutase (MnSOD) and cytochrome c signals in the STAT3 precipitation (Fig. 2a). Both serine-phosphorylated and total STAT3 co-immunoprecipitated with the presequence receptor Tom20 (translocase of the outer membrane 20), which is part of the mitochondrial import machinery, in rat LV protein extracts (Fig. 2c). Also, mitochondrial STAT3 co-immunoprecipitated with cyclophilin D, which is involved in the regulation of MPTP opening (Fig. 2d).

The submitochondrial localization of STAT3 was analyzed by western blot on rat LV SSM subfractionated into proteins of the intermembrane space, the inner and outer membrane, and the matrix. The matrix was not contaminated with marker proteins of the intermembrane space (cytochrome c), outer or inner membrane (VDAC or ATP synthase $\alpha$, respectively), but was positive for the marker protein cyclophilin D and for STAT3 (Figs. 3a, b). The presence of STAT3 in the mitochondrial matrix was confirmed by confocal laser scan microscopy (Appendix Fig. 11). When mitochondrial swelling was induced by incubating the mitochondria in hypoosmotic buffer, immunoreactivities of marker proteins of the mitochondrial intermembrane space (adenylate kinase 2 (AK2), cytochrome c) were reduced, whereas STAT3 signal intensity was unchanged (Fig. 3c). These data were confirmed by confocal laser scan microscopy showing that $80 \pm 3 \%$ of control mitochondria (as identified by MnSOD immunoreactivity, matrix marker protein) and $77 \pm 4 \%$ of swollen mitochondria were positive for STAT3, whereas the number of AK2-positive mitochondria was reduced from $38 \pm 4 \%$ in control mitochondria to $7 \pm 1 \%$ in swollen mitochondria $(n=3, p<0.05)$.

In order to characterize the function of mitochondrial STAT3, mitochondrial oxygen consumption was measured. Supporting prior data [44], ADP-stimulated respiration was reduced in STAT3-KO mitochondria using substrates for complex 1. However, no significant differences were found when using complex 2 substrates (Fig. 4), and when measuring complex 4-mediated respiration (in nmol $\mathrm{O}_{2} \mathrm{~min}^{-1} \mathrm{mg}^{-1}$ protein, STAT3-KO: $385 \pm 22, n=12$ vs. WT: $426 \pm 23, n=12, p=\mathrm{ns}$ ), or uncoupled respiration (STAT3-KO: $362 \pm 29, n=12$ vs. WT: $401 \pm 30$, $n=12, p=\mathrm{ns}$ ).

Incubation of isolated rat SSM with $100 \mu \mathrm{mol} / \mathrm{L}$ of the STAT3-specific inhibitor Stattic significantly decreased the 

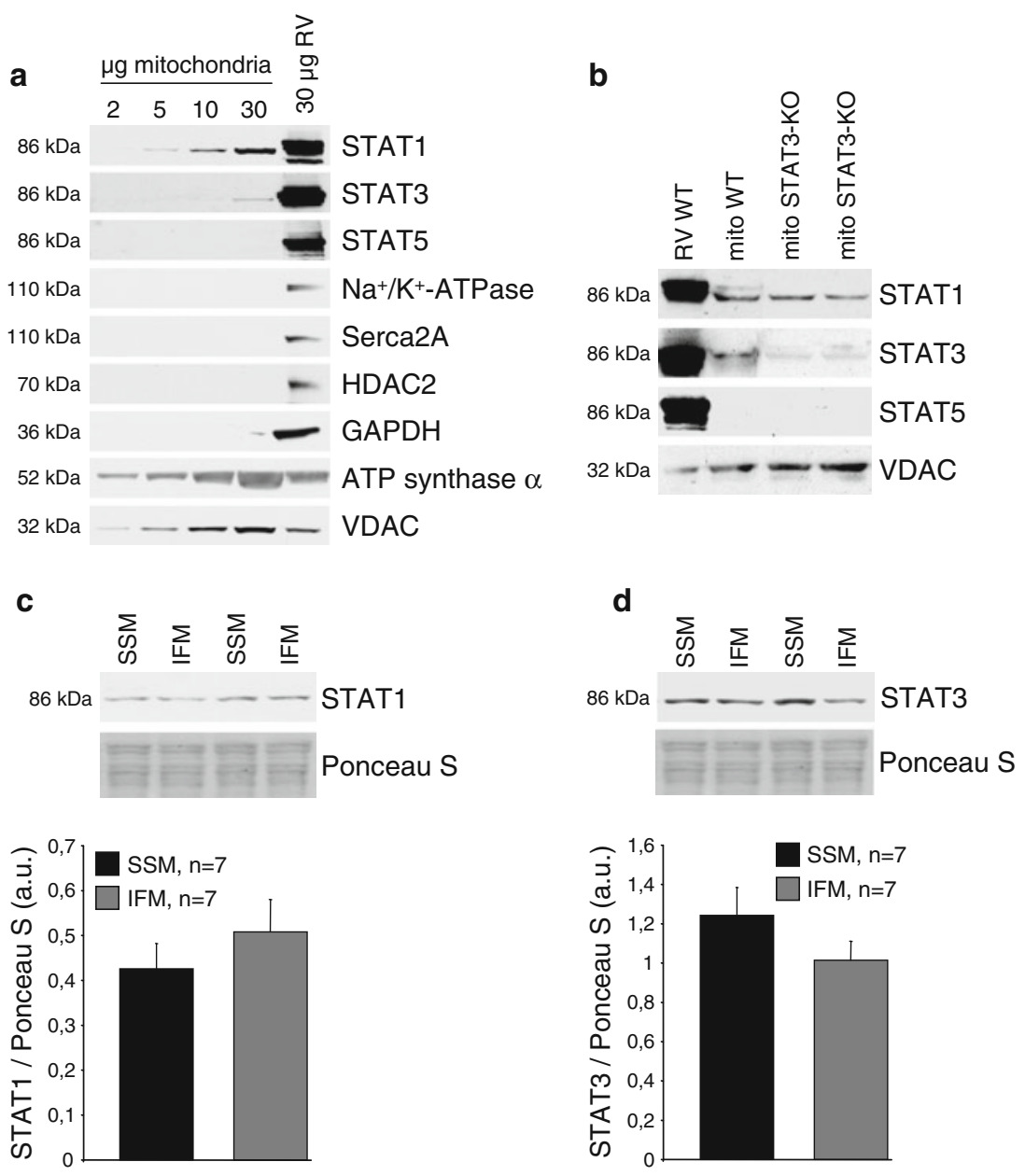

Fig. 1 STAT1 and 3 are present in LV mitochondria. a Western blot analysis was performed for STAT1, 3, and 5 and marker proteins of subcellular compartments $\left(\mathrm{Na}^{+} / \mathrm{K}^{+}\right.$-ATPase: sarcolemma, Serca2a: sarcoplasmic reticulum, HDAC2: nucleus, GAPDH: cytosol, ATP synthase $\alpha$ : mitochondria, VDAC: mitochondria) on rat total right ventricular $(\mathrm{RV})$ and different amounts of LV SSM protein extracts $(n=4)$. Western blots were exposed to X-ray films such that similar immunoreactivities were obtained for STAT1, 3, and 5 in RV protein extracts. b Western blot analysis was performed for STAT1, 3, 5, and the mitochondrial marker protein VDAC on $25 \mu \mathrm{g}$ total right

phosphorylation of STAT3 at Tyr705 and also tended to decrease the phosphorylation of STAT3 at Ser727 (Fig. 5a, b). Neither 100 nor $200 \mu \mathrm{mol} / \mathrm{L}$ of the STAT3-specific inhibitor Stattic affected mitochondrial autofluorescence or membrane potential, indicating that Stattic had no acute toxic effect on mitochondria (Appendix Fig. 12). Stattic did not impact on basal respiration using substrates for complex 1 or 2, but decreased ADP-stimulated respiration for complex 1 and-to a lesser extent-also for complex 2 (Fig. 5c, d).

Calcium-induced MPTP opening was measured by calcium green $5 \mathrm{~N}$. The calcium concentration which induced MPTP opening was similar in mitochondria from WT and ventricular (RV) and LV SSM proteins from WT and STAT3-KO mice $(n=4)$. c Western blot analysis was performed for STAT1 on SSM and IFM isolated from rat myocardium. Ponceau S staining is shown to demonstrate equal protein loading. The amount of STAT1 was normalized to Ponceau S staining of myocardial SSM and IFM. d Western blot analysis was performed for STAT3 on SSM and IFM isolated from rat myocardium. Ponceau $\mathrm{S}$ staining is shown to demonstrate equal protein loading. The amount of STAT3 was normalized to Ponceau S staining of myocardial SSM and IFM

STAT3-KO mice under basal conditions (no ADP and $\mathrm{MgCl}_{2}$ ) with complex 1 substrates (Fig. 6a). In contrast, in the presence of ADP and $\mathrm{MgCl}_{2}$, mimicking more closely the physiological situation, STAT3-KO mitochondria tolerated less calcium until MPTP opening occurred (Fig. 6b). Binding of cyclosporine A (CsA) to cyclophilin D delayed MPTP opening compared to control conditions, again, in the presence of ADP and $\mathrm{MgCl}_{2}$. The STAT3 inhibitor Stattic accelerated calcium-induced MPTP opening in rat mitochondria incubated with substrates for complex 1 even in the absence of ADP and $\mathrm{MgCl}_{2}$, and this effect was enhanced in the presence of ADP (Figs. 6c, d). CsA delayed MPTP opening to a lesser extent in Stattic-treated 

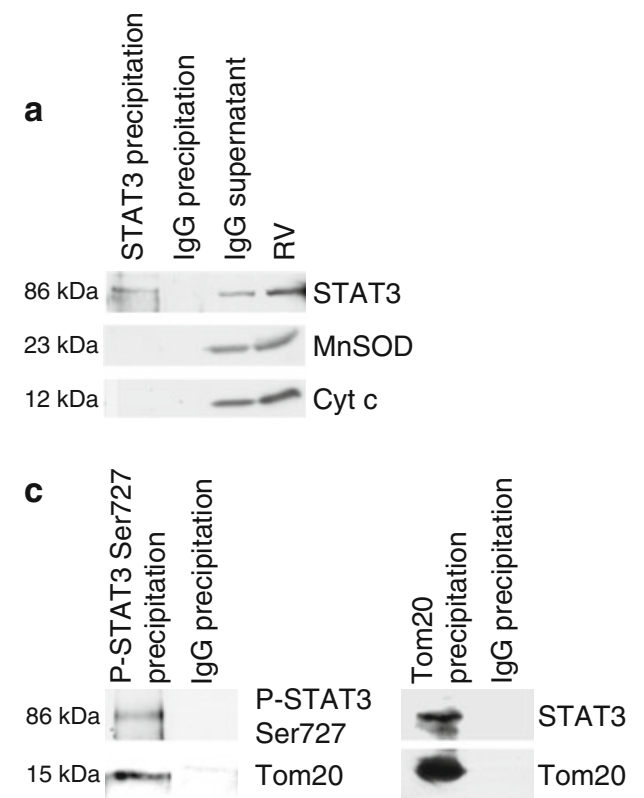

Fig. 2 Identification of mitochondrial proteins which co-immunoprecipitate with STAT3 and characterization of the dual mitochondrial STAT3 phosphorylation. Total STAT3 was immunoprecipitated from rat LV SSM. The specificity of the immunoprecipitation is indicated by the lack of STAT3 in the IgG precipitation as well as by the lack of MnSOD and cytochrome c (Cyt C) immunoreactivities (a).
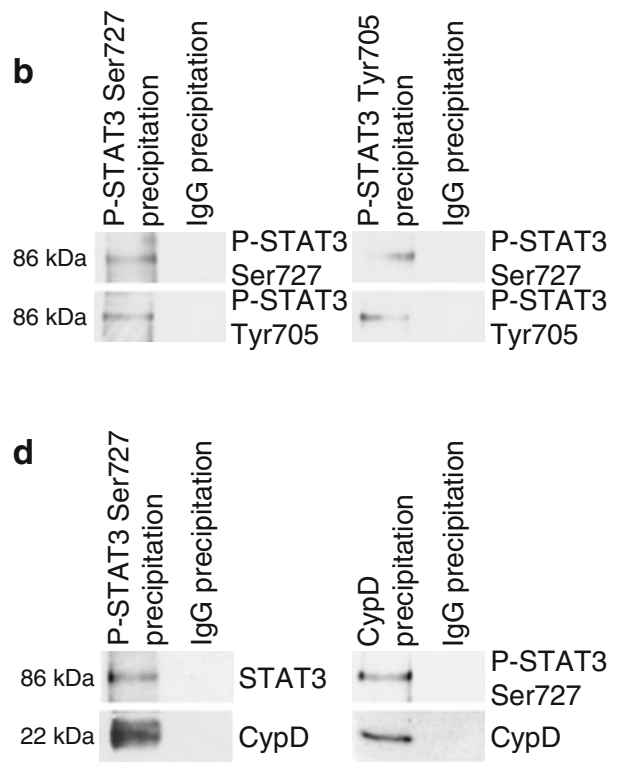

Phosphorylated STAT3 [Ser727 and Tyr705 (b)], as well as Tom20 (c) or cyclophilin $\mathrm{D}(\mathrm{CypD}, \mathbf{d})$ were immunoprecipitated from rat SSM proteins. Rabbit IgGs were used as negative control. Western blot analysis was performed for total or phosphorylated STAT3 (Ser727 and Tyr705), Tom20, and CypD ( $n=3-6)$

a

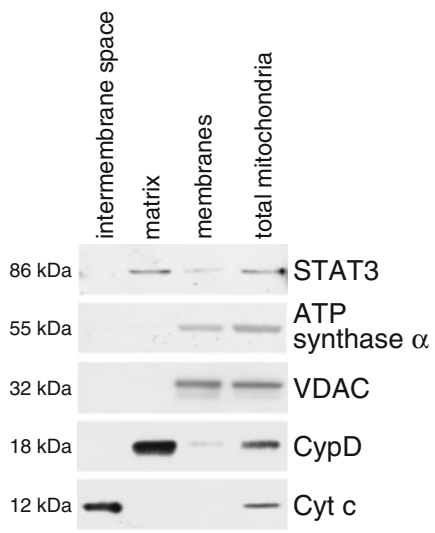

b

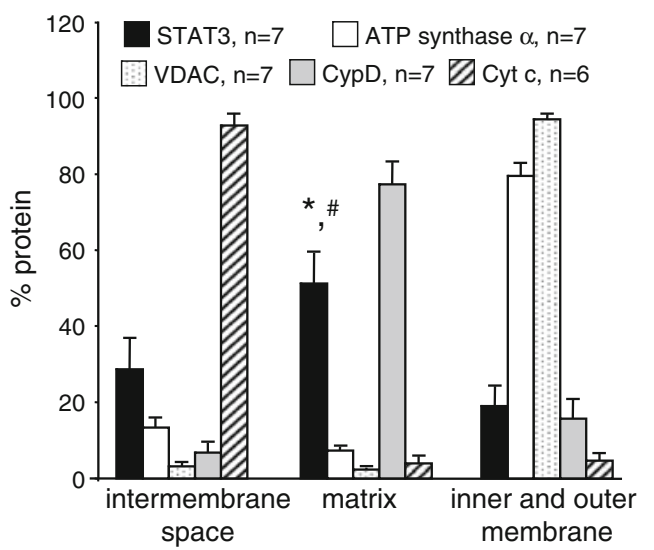

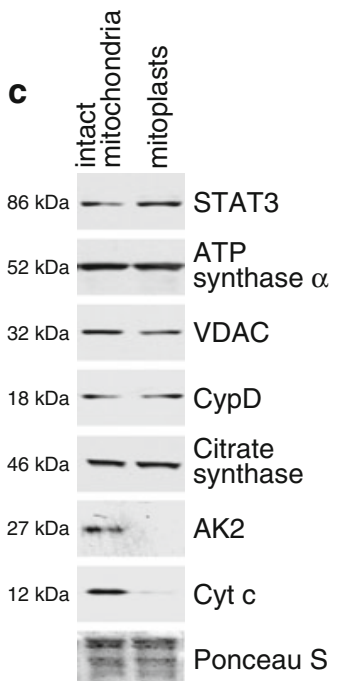

Fig. 3 STAT3 is present in the mitochondrial matrix. a Rat LV SSM were subfractionated into the proteins of the intermembrane space, the matrix, and the inner and outer membrane (membranes). Western blot analysis was performed for STAT3 and marker proteins of different submitochondrial compartments (intermembrane space: Cyt c, matrix: CypD, outer membrane: VDAC, inner membrane: ATP synthase $\alpha$ ). Total mitochondrial protein extracts were used as control. b Bar graphs representing the percentage of protein in mitochondrial compartments. The total amount of pixels from each subfractionation protocol was set as $100 \%$. ${ }^{*}, p<0.05$ for STAT3 immunoreactivity

than in DMSO-treated mitochondria. In STAT3-KO mitochondria, Stattic did not alter calcium-induced MPTP opening in the absence of ADP (in nmol $\mathrm{CaCl}_{2} / \mathrm{mg}$ protein, in the matrix versus the intermembrane space and the inner and outer membrane, respectively. c Rat LV SSM were incubated in hypoosmotic buffer to induce mitochondrial swelling and subsequently characterized by western blot analysis. Swelling induces loss of marker proteins for the intermembrane space (adenylate kinase 2, $\mathrm{AK} 2$, and cytochrome $\mathrm{C}$, Cyt c), whereas immunoreactivities for membrane proteins (ATP synthase $\alpha$, inner membrane) or VDAC (outer membrane), matrix proteins [citrate synthase, cyclophilin D (CypD)] as well as STAT3 immunoreactivity were unchanged

DMSO: $\quad 726.5 \pm 16.1 ; \quad$ Stattic: $713.5 \pm 24.0, \quad n=6$, $p=\mathrm{ns})$, thereby excluding unspecific effects of Stattic on MPTP opening. 
Fig. 4 Genetic reduction of STAT3 decreases mitochondrial ADP-stimulated oxygen consumption. Oxygen consumption of WT $(n=12)$ and STAT3-KO $(n=12)$ SSM was measured under basal conditions and after addition of $40 \mu \mathrm{mol} / \mathrm{L}$ ADP using glutamate/malate as substrates for complex 1 (a) or succinate with rotenone as substrate for complex 2 (b)
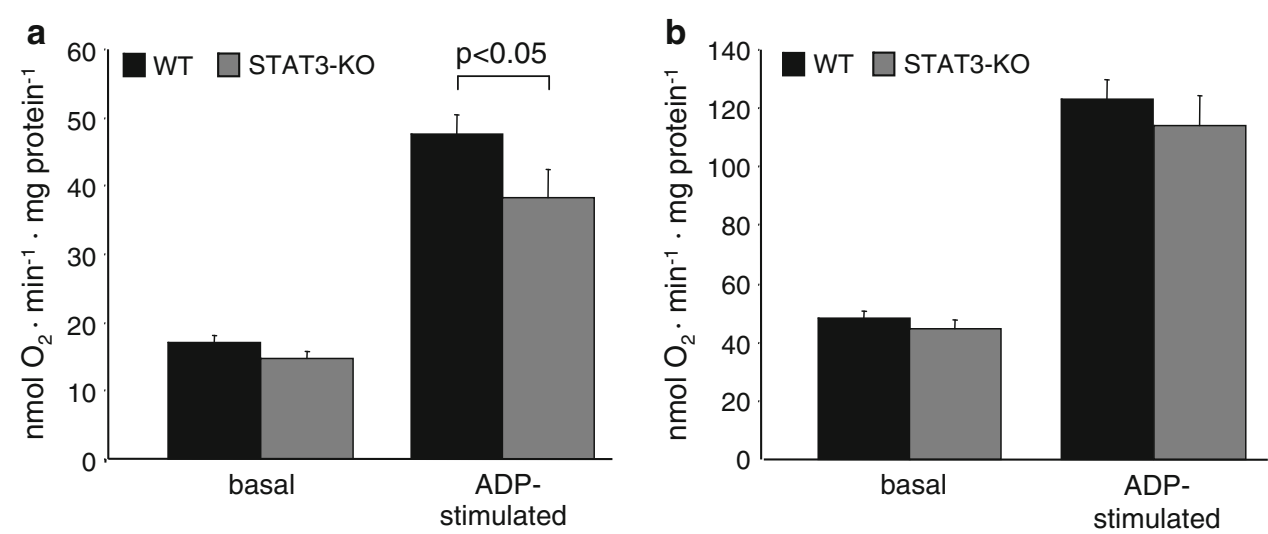

a

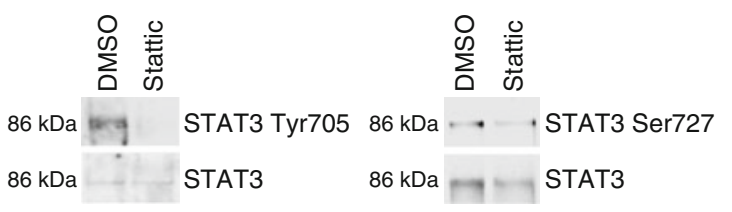

b
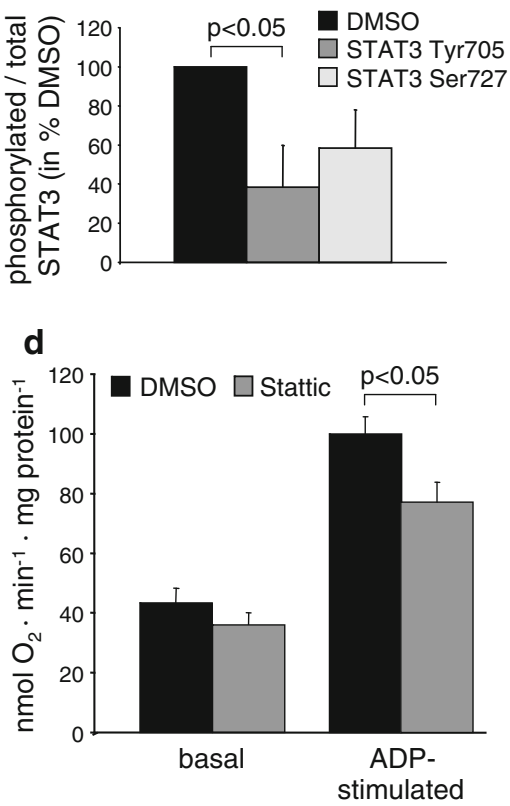

Fig. 5 Pharmacological inhibition of STAT3 decreases mitochondrial ADP-stimulated oxygen consumption. a Total STAT3 was immunoprecipitated from purified rat LV SSM which were incubated with $100 \mu \mathrm{mol} / \mathrm{L}$ Stattic or DMSO. Western blot analysis was performed for STAT3 phosphorylated at tyrosine 705, serine 727 or total STAT3 $(n=4)$. The ratio of phosphorylated over total STAT3

Using substrates for complex 2, MPTP opening in the presence of ADP and $\mathrm{MgCl}_{2}$ was induced at similar calcium concentrations in WT and STAT3-KO mitochondria (in $\mathrm{nmol} \mathrm{CaCl}_{2} / \mathrm{mg}$ protein, WT: $1,164 \pm 47, n=7$ vs. STAT3-KO: 1,264 $\pm 56, \mathrm{n}=7, p=\mathrm{ns}$ ). However, Stattic treatment of rat mitochondria again accelerated MPTP opening (in $\mathrm{nmol} \mathrm{CaCl}_{2} / \mathrm{mg}$ protein, DMSO: $1,357 \pm 67$, $n=7$ vs. Stattic: $629 \pm 39, n=7, p<0.05$ ).

Since STAT3 co-immunoprecipitated with cyclophilin D and cyclophilin D inhibition with CsA delayed MPTP more effectively in control than in STAT3-KO or Stattictreated mitochondria, we studied the effects of CsA on ischemia/reperfusion injury in WT and STAT3-KO in Stattic-treated mitochondria is shown in percentage of DMSOtreated control mitochondria (b). Oxygen consumption was measured under basal conditions and after addition of $40 \mu \mathrm{M}$ ADP to rat LV SSM incubated for $1 \mathrm{~h}$ at $4^{\circ} \mathrm{C}$ with DMSO or $100 \mu \mathrm{mol} / \mathrm{L}$ Stattic using glutamate/malate as substrates for complex $1(\mathbf{c}, n=6)$ or succinate with rotenone as substrate for complex $2(\mathbf{d}, n=6)$

hearts in vivo. The area at risk (as percentage of the LV) was not different between groups (WT $+\mathrm{NaCl}$ : $25.5 \pm 1.8 \%, n=7$; STAT3 $\mathrm{KO}+\mathrm{NaCl}: 31.1 \pm 1.5 \%$, $n=7 ; \quad$ WT + CsA: $\quad 25.4 \pm 2.2, \quad n=10 ; \quad$ STAT3 $\mathrm{KO}+\mathrm{CsA}: 25.6 \pm 1.1, n=7, p=\mathrm{ns})$. CsA reduced infarct size after $30 \mathrm{~min}$ ischemia and $120 \mathrm{~min}$ reperfusion to a similar extent in WT and in STAT3-KO hearts in vivo (Fig. 7). Comparison with historical data [2] demonstrated that CsA and ischemic postconditioning induced infarct size reduction of similar magnitude in WT mice, whereas the cardioprotection by ischemic postconditioning, but not by CsA was lost in STAT3-KO mice. 
Fig. 6 Genetic reduction and pharmacological inhibition of STAT3 enhance calciuminduced MPTP opening. The calcium concentration at which opening of the MPTP occurred (increase in calcium green $5 \mathrm{~N}$ fluorescence) was calculated in STAT3-KO or WT mouse SSM in the presence or absence of CsA using a substrates for complex 1 without ADP and $\mathrm{MgCl}_{2}$ (WT: $n=7$, STAT3KO: $n=9)$ or b with ADP and $\mathrm{MgCl}_{2}$ (WT: $n=10$, STAT3KO: $n=11$ ), and in Stattic- or DMSO-treated rat SSM in the presence or absence of CsA using c substrates for complex 1 without $\mathrm{ADP}$ and $\mathrm{MgCl}_{2}$ $(n=9)$ or $\mathbf{d}$ with ADP and $\mathrm{MgCl}_{2}(n=7)$
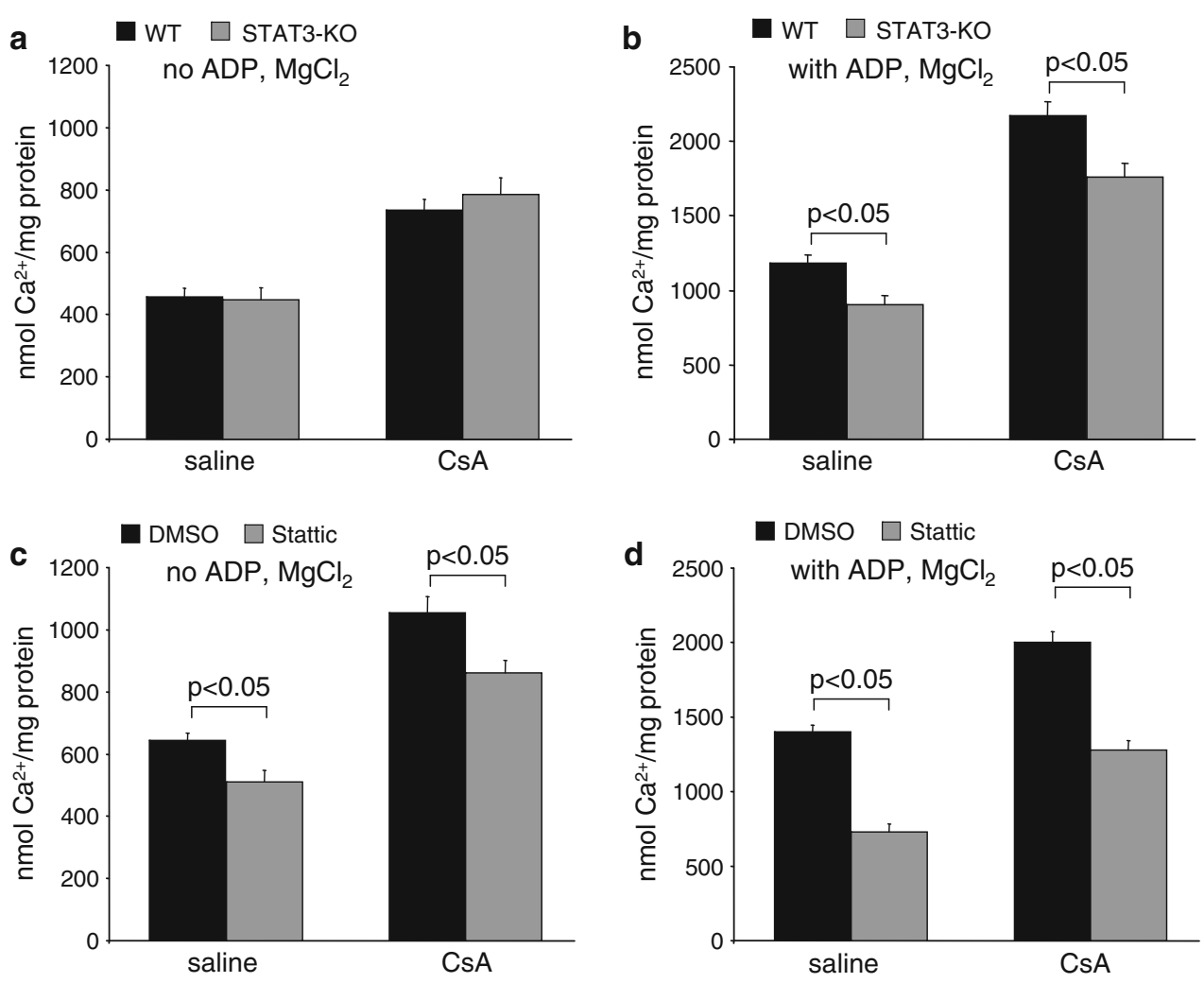

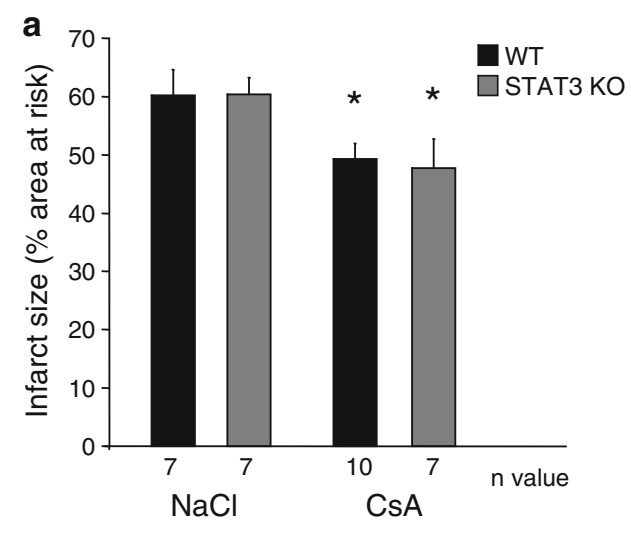

Fig. 7 STAT3 is important for infarct size reduction by ischemic postconditioning, but not for pharmacological postconditioning with cyclosporine A (CsA). a Infarct size (in \% of the area at risk) was determined in WT and STAT3-KO mice undergoing $30 \mathrm{~min}$ ischemia and $120 \mathrm{~min}$ reperfusion. $10 \mathrm{mg} / \mathrm{kg} \mathrm{CsA}$ or $0.9 \% \mathrm{NaCl}$ solution were

We have reported a decrease of STAT3 in RV total protein extracts from aged WT mice before [2, 5]. In the present study, a reduced STAT3 protein level was also seen in mitochondria isolated from LV tissue of aged mice (Fig. 8). As positive control served the decreased level of connexin 43 in aged mitochondria, which we have also described before [5]. In contrast, the mitochondrial content of ATP synthase $\alpha$ was not reduced with age.

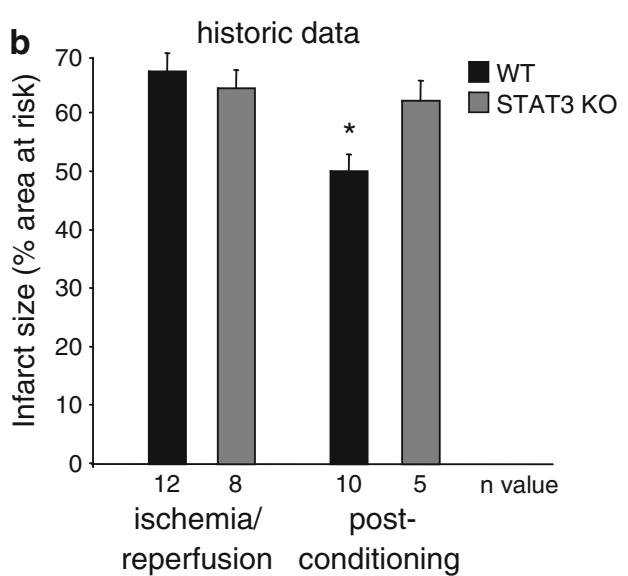

administered intravenously $5 \mathrm{~min}$ before reperfusion, $* p<0.05$. b Historic data [2] showing that ischemic postconditioning by 3 cycles of $10 \mathrm{~s}$ ischemia and reperfusion each at the onset of reperfusion reduced infarct size after $30 \mathrm{~min}$ ischemia and $120 \mathrm{~min}$ reperfusion in WT, but not in STAT3-KO mice, $* p<0.05$

\section{Discussion}

The present study demonstrates the presence of STAT1 and STAT3, but not of STAT5 in cardiomyocyte mitochondria. Our data confirm the previously described localization of STAT3 in cardiomyocyte mitochondria [44] and extend these prior data in that the mitochondrial localization of STAT proteins is not restricted to STAT3 but also true for 
Fig. 8 Mitochondrial STAT3 content is reduced in aged mouse hearts. Western blot analysis was performed for STAT3 on LV SSM from young ( 8 weeks) and aged (21 months) wildtype mice. As positive control served the reduced mitochondrial content of connexin 43 and as negative control the unchanged protein content of ATP synthase $\alpha$
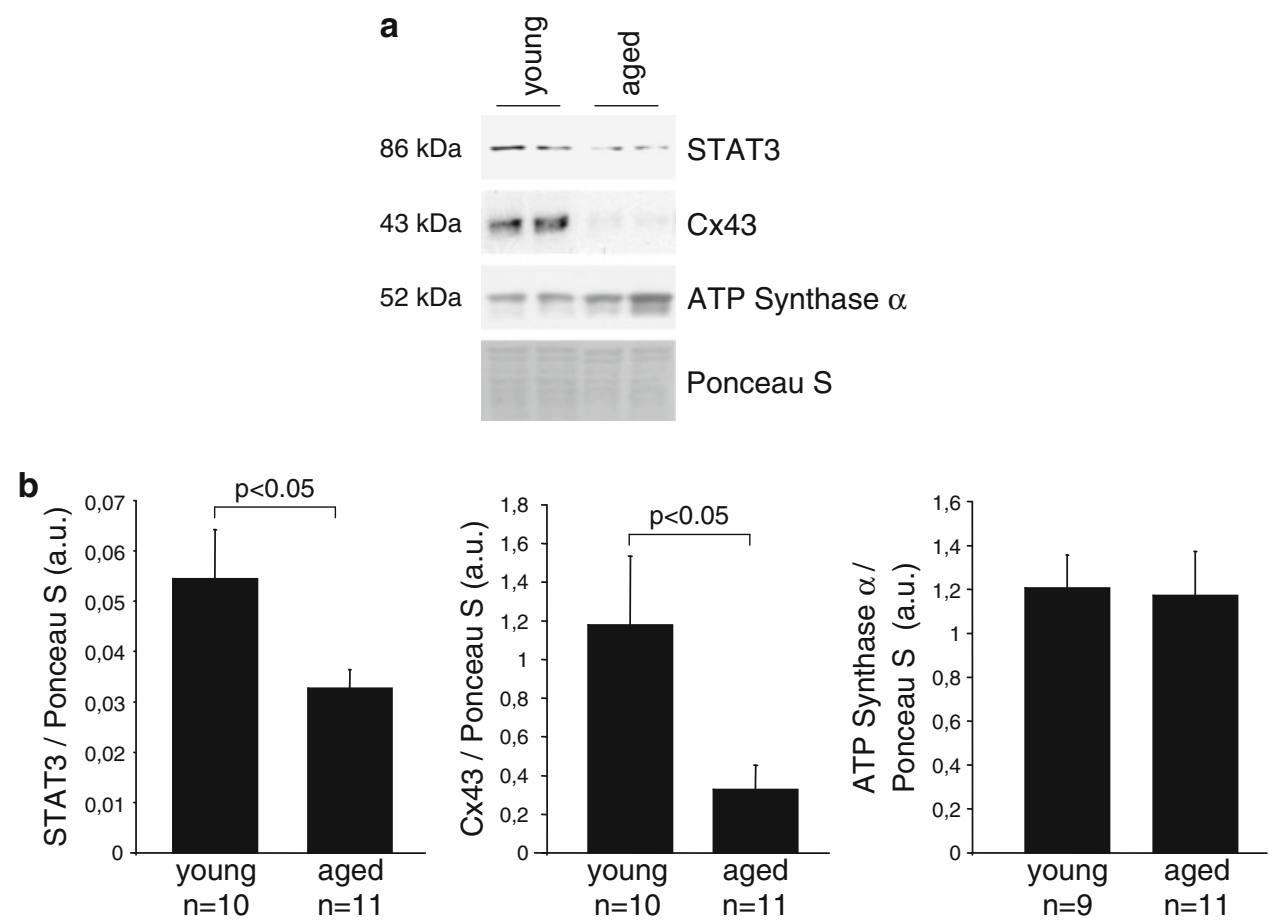

STAT1. The lack of STAT5 in cardiomyocyte mitochondria under basal conditions does not exclude a possible mitochondrial import of STAT5 following ischemia/ reperfusion; alternatively, the STAT5-mediated cardioprotection by ischemic preconditioning [46] works independently from mitochondrial signal transduction cascades.

In the present study, STAT1 and STAT3 were found in SSM and IFM, mitochondrial populations which differ in their protein composition as well as in their respiratory function and calcium retention capacity [7, 29, 35]. Therefore, the opposing roles of STAT1 and STAT3 in myocardial ischemia/reperfusion-STAT1 is associated with enhanced and STAT3 with reduced cardiomyocyte death after ischemia/reperfusion (for review see [4]) cannot be attributed to diverging localizations in mitochondrial subpopulations under physiological conditions. However, whether or not the distribution of STAT1 and STAT3 in mitochondrial subpopulations is changed after ischemia/reperfusion is currently unknown. The residual STAT3 immunoreactivity detected by western blot analysis in mitochondria from STAT3-KO hearts is presumably due to the presence of mitochondria from endothelial cells or smooth muscle cells-i.e. other STAT3 expressing cell types in the heart which are not affected by the cardiomyocyte-specific STAT3 knockout, but are included in the mitochondrial preparation.

STAT3 is encoded in the nucleus and therefore has to be imported into the mitochondria. Both phosphorylated and total STAT3 co-immunoprecipitated with the mitochondrial presequence receptor Tom20, suggesting an import of STAT3 via a Tom20-dependent pathway.

The transcriptional activity of STAT3 is regulated by post-translational modification, i.e. phosphorylation and dephosphorylation. Two major phosphorylation sites are known: phosphorylation at tyrosine 705 via Janus kinases which induces dimerization and translocation into the nucleus, and phosphorylation at serine 727 which plays a role in modulating transcriptional activity [25]. Mitochondrial STAT3 was phosphorylated at both tyrosine 705 and serine 727 . The phosphorylation of STAT3 at serine 727 appears to be more pronounced. Our data do not allow to distinguish whether or not STAT3 is phosphorylated prior to its import or within the mitochondria. Subfractionated mitochondria were characterized to identify the submitochondrial localization of STAT3, and STAT3 was predominantly detected in the mitochondrial matrix. However, the degree of phosphorylation of STAT3 in different mitochondrial compartments remains unclear. Our data are in accordance to and extend the data by Wegrzyn et al. [44], who have previously excluded the presence of STAT3 in the outer membrane of liver mitochondria. STAT3 as a transcriptional activator in the mitochondrial matrix may influence the transcription of genes encoded in the chondriome. However, Wegrzyn et al. [44] found no differences in the transcript levels of mitochondrially encoded genes in wildtype and STAT3-deficient pro-B cells. These results suggest that mitochondrial STAT3 serves a function different from transcriptional activation. Previous data 
propose that the serine 727 phosphorylation is important for the effect of STAT3 on mitochondrial respiratory complex 1 and 2 activities [27, 44].

In the present study, mitochondria isolated from STAT3-KO hearts had reduced ADP-stimulated complex 1 respiration compared to mitochondria isolated from WT hearts, confirming previous results [44]. However, when measuring complex 2- or complex 4-mediated respiration and uncoupled respiration, only a trend towards a decreased ADP-stimulated respiration was detected. STAT3-deficiency was associated with decreased complex 5 activity and reduced ATP contents in H-RasV12-transformed cells [12]; however, the present study did not characterize complex 5 activity and ATP-production of isolated cardiomyocyte mitochondria.

The investigation of mitochondria isolated from STAT3-KO hearts does not allow to distinguish whether the effects of STAT3 on respiration are caused directly by mitochondrial STAT3 or indirectly by changing the transcription of nuclear-encoded mitochondrial target genes. We therefore also studied respiration in rat mitochondria, which were incubated with the STAT3-specific inhibitor Stattic. The concentration of $100 \mu \mathrm{mol} / \mathrm{L}$ Stattic has previously been shown to effectively reduce the tyrosine phosphorylation of STAT3 in isolated mouse hearts [11]. In the present study, Stattic also decreased mitochondrial STAT3 tyrosine 705 and tended to reduce STAT3 serine 727 phosphorylation. In Stattic-treated mitochondria, ADP-stimulated complex 1 respiration was reduced to about $50 \%$ of control. Using complex 2 substrates, Stattic reduced ADP-stimulated respiration again to a lesser extent than with complex 1 substrates. Taken together, genetic reduction and pharmacological inhibition of STAT3 did not alter basal respiration, but decreased ADPstimulated respiration, notably that of complex 1 . Whereas STAT3 has been demonstrated to be associated with complex 1 and possibly with complex 2 [44], a recent study calculated the ratio of complexes 1 and 2 over STAT3 to be in the order of $10^{5}$ [31], making a direct protein-protein interaction between STAT3 and complexes 1 and 2 unlikely. However, STAT3 may influence respiration indirectly via modulation of the activity of protein kinases, e.g. by facilitating the docking of kinases to target proteins [30].

The MPTP, a voltage-dependent, high conductance mitochondrial membrane channel, opens when exposed to high concentrations of reactive oxygen species and calcium at normal intracellular $\mathrm{pH}$. The well-established inhibitory effects of ADP and $\mathrm{MgCl}_{2}$ on calcium-induced MPTP opening $[9,34]$ were confirmed in the present study, since the amounts of calcium needed to induce MPTP opening were higher in the presence of ADP and $\mathrm{MgCl}_{2}$. Opening of the MPTP releases cytochrome $\mathrm{c}$ and induces cardiomyocyte apoptosis and necrosis. Inhibition of MPTP opening is important for cardiomyocyte survival and the cardioprotection by ischemic pre- and postconditioning $[8,15,19,21]$. Calcium-induced MPTP opening occurred at lower calcium concentrations in STAT3-KO mitochondria than in WT mitochondria respiring on complex 1 only in the presence of ADP and $\mathrm{MgCl}_{2}$. The more pronounced MPTP opening of mitochondria respiring on complex 1 with ADP and $\mathrm{MgCl}_{2}$ compared to basal conditions was also confirmed in Stattic-treated rat mitochondria. Stattic enhanced calcium-induced MPTP opening already under basal conditions in rat mitochondria, but not in mouse STAT3-KO mitochondria. Such accelerated MPTP opening in STAT3-KO mitochondria, especially in the presence of ADP and $\mathrm{MgCl}_{2}$, may contribute to the loss of endogenous cardioprotection in STAT3-deficient mice. Whether or not the impact of STAT3 on MPTP opening is due to changes in the mitochondrial membrane potential is unknown at present.

The more pronounced effect of Stattic on respiration and MPTP opening compared to that of STAT3-KO mitochondria may be due to the fact that Stattic inhibits all STAT3-containing mitochondria, whereas in mitochondrial preparations from mice with a cardiomyocyte-specific deletion of STAT3, a certain amount of STAT3 containing mitochondria from endothelial cells, vascular smooth muscle cells or invading blood cells is still present.

STAT3 co-immunoprecipitated with cyclophilin D, a matrix protein which facilitates MPTP opening when bound to the inner mitochondrial membrane [9]. CsA prevents binding of cyclophilin D to the inner mitochondrial membrane, thereby delays MPTP opening and contributes to infarct size reduction after ischemia/reperfusion injury in animals [1, 16, 21, 40] and man [32]. In the present study, CsA delayed calcium-induced MPTP opening in isolated mitochondria; however, this delay was more pronounced in control mitochondria than in STAT3-KO or Stattic-treated mitochondria. Whereas these in vitro data might suggest that infarct size reduction by CsA in vivo might be more pronounced in WT than in STAT3-KO mice, the present study demonstrated in fact a similar infarct size reduction by CsA in both genotypes. The magnitude of infarct size reduction by CsA was comparable to that achieved by ischemic postconditioning in WT mice [2]. Therefore, inhibition of cyclophilin D by CsA appears to be rather downstream of STAT3 in the signaling cascade of cardioprotection, or STAT3 and CsA interaction with different domains of cyclophilin D. The reduction of reperfusion injury by maneuvers such as ischemic postconditioning is dependent on STAT3 [2, 22], whereas the reduction of reperfusion injury by inhibition of the STAT3 co-precipitating protein cyclophilin D is apparently downstream of STAT3. 
Limitations

The detection of total and phosphorylated STAT1 and STAT3 in mitochondria by western blot analysis and confocal laser scan microscopy is dependent on the affinities of the respective antibodies towards their target epitopes. Therefore, an accurate quantification of the amounts of total mitochondrial STAT1 and STAT3 is not possible without using purified proteins as standard. Whereas our immunoprecipitation and confocal laser scan microscopy data suggest that the phosphorylation of STAT3 is more prominent at Ser727 than at Tyr705, we cannot quantify the ratio of serine over tyrosine phosphorylated mitochondrial STAT3. The STAT3 inhibitor Stattic has been described to block the phosphorylation of STAT3 at Tyr705, and we confirmed this result in isolated mitochondria. However, the phosphorylation of STAT3 at Ser727 also tended to be decreased by Stattic. We can therefore not distinguish whether the decreased APD-stimulated respiration and the enhanced calcium-induced MPTP opening in Stattic-treated mitochondria are due to the inhibition of the STAT3 phosphorylation at residue Ser727, Tyr705 or both. One could speculate that the enhanced calcium-induced MPTP opening in STAT3-deficient mitochondria would be paralleled by enhanced infarct size in STAT3 KO mice after ischemia/ reperfusion injury in vivo. However, similar infarct sizes were measured in WT and STAT3 KO mice. In contrast to the unaltered infarct size with ischemia/reperfusion per se, endogenous cardioprotection by ischemic pre- and postconditioning was abolished in STAT3 KO mice. These findings suggest that STAT3 deficiency can be compensated during ischemia/reperfusion, but that these compensatory mechanisms are not sufficient to mediate endogenous cardioprotection by ischemic pre- and postconditioning.

\section{Potential clinical relevance}

The reduced mitochondrial STAT3 level in aged hearts may be important for the loss of cardioprotection in aged mice $[2,5,6]$. Apart from aging, STAT3 expression is decreased in rats with diabetes [13] and in patients with end-stage heart failure [33], pathophysiological conditions also associated with loss of endogenous cardioprotection. The targeting of STAT3 to the mitochondria promotes Rasdependent oncogenic transformation [12]. However, the present data also point to beneficial roles of mitochondrial STAT3 for maintaining mitochondrial function and thereby enhancing cell survival. Mitochondrial STAT3 possibly contributes to cardioprotection by augmentation of ADPstimulated respiration and inhibition of MPTP opening, but not through interaction with CsA binding to cyclophilin D. Our data suggest that patients with reduced contents of
STAT3 would benefit from CsA therapy in acute myocardial infarction.

Taken together, STAT1 and STAT3 are localized in the matrix of cardiomyocyte mitochondria. Mitochondrial STAT3 stimulates respiration and inhibits calcium-induced MPTP opening, thereby potentially contributing to cardioprotection.

Acknowledgments We thank Dr. Sabine Stahlhofen and Astrid Büchert, Elvira Ungefug, Ina Konietzka, Anita van de Sand, and Petra Gres for excellent technical assistance. K.B. (BO 2955/1-1) and G.H. (He1320/18-1) are recipients of grants from the Deutsche Forschungsgemeinschaft.

Conflict of interest None.

\section{Appendix 1: Pharmacological inhibition of STAT3}

To exclude acute toxic effects of Stattic (STAT3 inhibitory compund, 6-Nitrobenzo[b]thiophene 1,1-dioxide), mitochondrial $\mathrm{NAD}(\mathrm{P}) \mathrm{H}$ autofluorescence and membrane potential were measured of $0.5 \mathrm{mg} / \mathrm{mL}$ rat LV SSM. The effect of Stattic or DMSO as vehicle, respectively, on the mitochondrial $\mathrm{NAD}(\mathrm{P}) \mathrm{H}$ autofluorescence (excitation $340 \mathrm{~nm}$, emission $460 \mathrm{~nm}$ ) or membrane potential $(10 \mathrm{nmol} / \mathrm{L}$ of the potentiometric dye rhodamine 123 , excitation $503 \mathrm{~nm}$; emission $535 \mathrm{~nm}$ ) was measured at $25^{\circ} \mathrm{C}$ in incubation buffer [containing in mmol/L: $125 \mathrm{KCl}$, 10 Tris/MOPS (3-( $N$-(morpholino)propanesulfonic acid), $1.2 \mathrm{Pi} /$ Tris, $1.2 \mathrm{MgCl}_{2}, 0.02$ EGTA, 5 glutamate, 2.5 malate, $\mathrm{pH}$ 7.4] in a Cary Eclipse spectrophotometer (Varian, Mulgrave, Australia). The difference between the fluorescence intensity $1 \mathrm{~min}$ prior and $1 \mathrm{~min}$ after the addition of DMSO, $100 \mu \mathrm{mol} / \mathrm{L}$ Stattic or $200 \mu \mathrm{mol} / \mathrm{L}$ Stattic, respectively, was quantified. To induce collapse of the mitochondrial membrane potential, $100 \mathrm{nmol} / \mathrm{L}$ FCCP was added, and the fluorescence intensity was calculated 1 min after FCCP administration $(n=5)$.

\section{Appendix 2: Confocal laser scan microscopy}

For mitochondrial subfractionation, rat $\mathrm{LV}$ mitochondria were incubated in isolation buffer with $600 \mu \mathrm{mol} / \mathrm{L}$ digitonin for $10 \mathrm{~min}$ on ice. Mitochondria were collected by centrifugation and subsequently incubated with $150 \mathrm{mmol} /$ $\mathrm{L} \mathrm{KCl}$ for $10 \mathrm{~min}$ on ice. Again, mitochondria were collected by centrifugation and resuspended in phosphatebuffered saline (PBS). Subfractionation of mitochondria was performed, as previously described [36].

Purified rat, mouse WT, or STAT3-KO LV mitochondria (intact or treated with digitonin and $\mathrm{KCl}$ ) were 
incubated with anti-STAT3 Tyr705, anti-STAT3 Ser727, anti-STAT3, anti-ATP synthase $\alpha$, anti-cytochrome $\mathrm{c}$, or anti-cyclophilin $\mathrm{D}$ antibodies for $1 \mathrm{~h}$ at $25^{\circ} \mathrm{C}$ with gentle agitation and, after washing, with the respective secondary antibodies. Mitochondria were mounted with Vectashield and were examined by confocal laser scan microscopy
Fig. 9 Total and

phosphorylated STAT3 are present in mouse left ventricular (LV) mitochondria. a LV subsarcolemmal mitochondria $(\mathrm{SSM})$ of WT $(n=6)$ and STAT3-KO mice $(n=8)$ were stained with antibodies against STAT3 (green) and the mitochondrial marker cyclophilin D (red) and were analyzed by confocal laser scan microscopy. Overlay pixels are shown in yellow. Whereas in SSM isolated from the LV of WT mice about $91 \%$ (347 mitochondria counted) of the ATP synthase positive mitochondria were also positive for STAT3, only $13 \%$ (248 mitochondria counted) of the SSM had immunoreactivity for STAT3 in STAT3-KO mice. b SSM from the LV of WT $(n=4)$ and STAT3-KO mice $(n=4)$ were stained with antibodies against STAT3 phosphorylated at Ser727 (green) and the mitochondrial marker cyclophilin D (red) and were analyzed by confocal laser scan microscopy. Overlay pixels are shown in yellow. c SSM from the LV of WT $(n=4)$ and STAT3-KO mice $(n=4)$ were stained with antibodies against STAT3 phosphorylated at Tyr705 (green) and the mitochondrial marker cyclophilin D (red) and were analyzed by confocal laser scan microscopy. Overlay pixels are shown in yellow
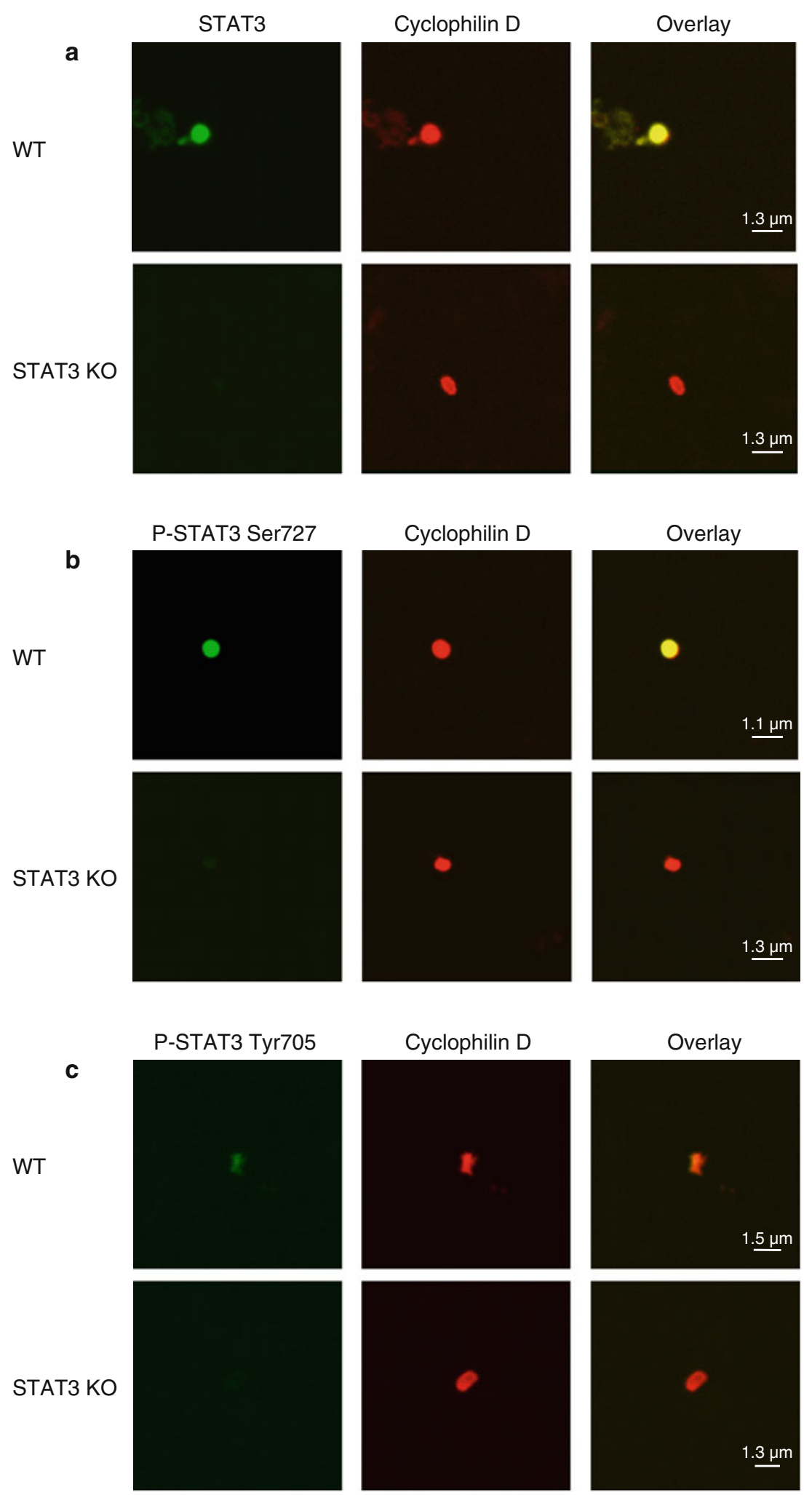

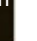


a
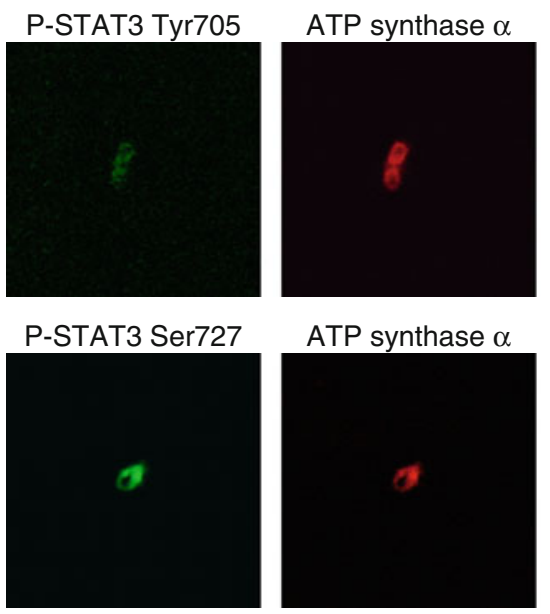

ATP synthase $\alpha$

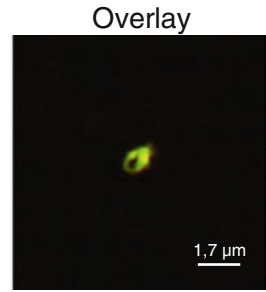

b
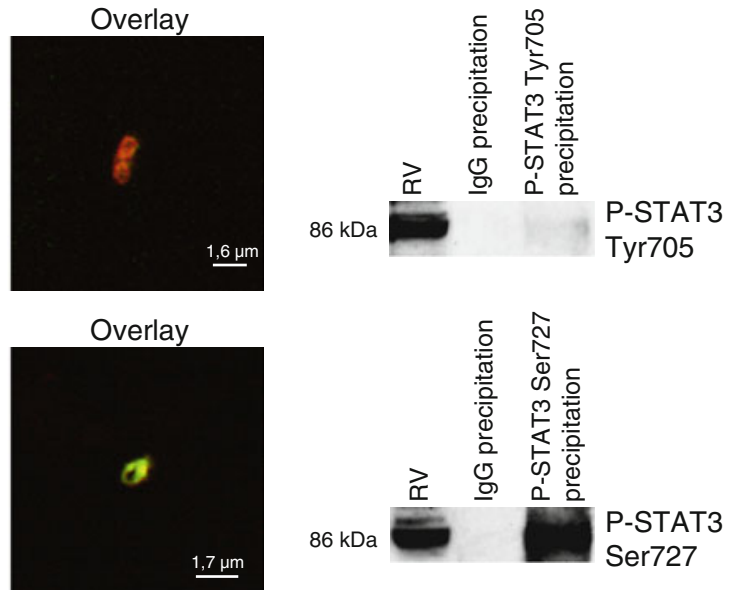

Fig. 10 Mitochondrial STAT3 is phosphorylated. a Subsarcolemmal mitochondria (SSM) from the rat LV were stained with antibodies against STAT3 phosphorylated at Tyr705 or Ser727 (green) and the mitochondrial marker ATP synthase $\alpha$ (red) and were analyzed by confocal laser scan microscopy $(n=4)$. Overlay pixels are shown in yellow. b STAT3 phosphorylated at Tyr705 or Ser727 was immunoprecipitated from rat SSM proteins. IgGs were used as negative control. Western blot analysis on immunoprecipitated proteins was performed for serine or tyrosine phosphorylated STAT3 $(n=4)$. To compare signal intensities for STAT3 phosphorylated at Tyr705 or Ser727, western blots were exposed to X-ray films such that the signal intensities for phosphorylated STAT3 in total right ventricular $(R V)$ protein extracts were similar
Fig. 11 STAT3 is present in the matrix of left ventricular (LV) subsarcolemmal mitochondria (SSM). a LV rat SSM (untreated or treated with digitonin and $\mathrm{KCl}$ in order to remove proteins from the outer membrane and the intermembrane space) were stained with antibodies against cytochrome c (intermembrane space, red) and cyclophilin D (matrix, green), and were analyzed by confocal laser scan microscopy. Overlay pixels are shown in yellow $(n=3)$.

b Untreated or digitonin/KCltreated SSM were stained with antibodies against STAT3 (red) and cyclophilin D (green) and were analyzed by confocal laser scan microscopy. Overlay pixels are shown in yellow $(n=3)$
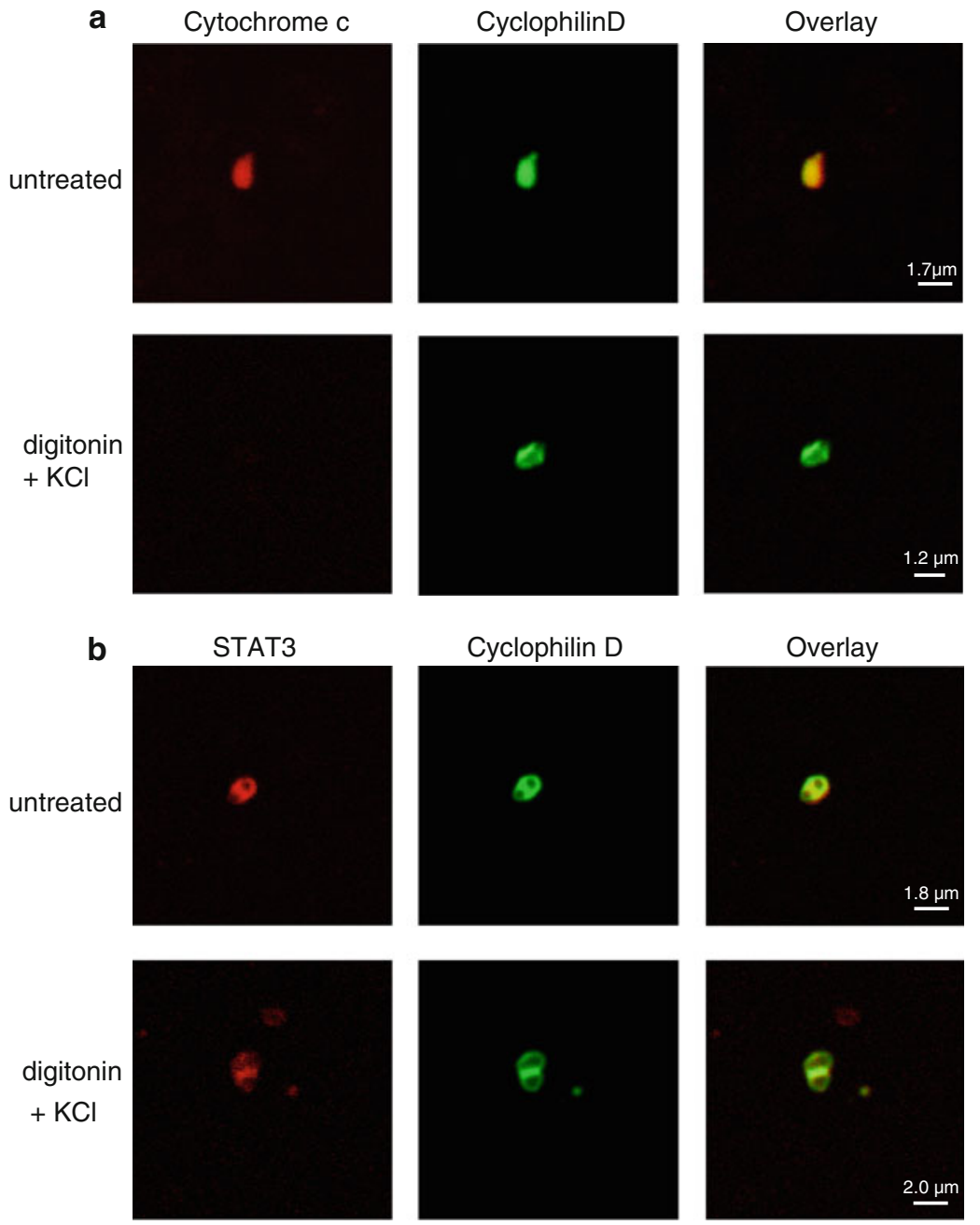

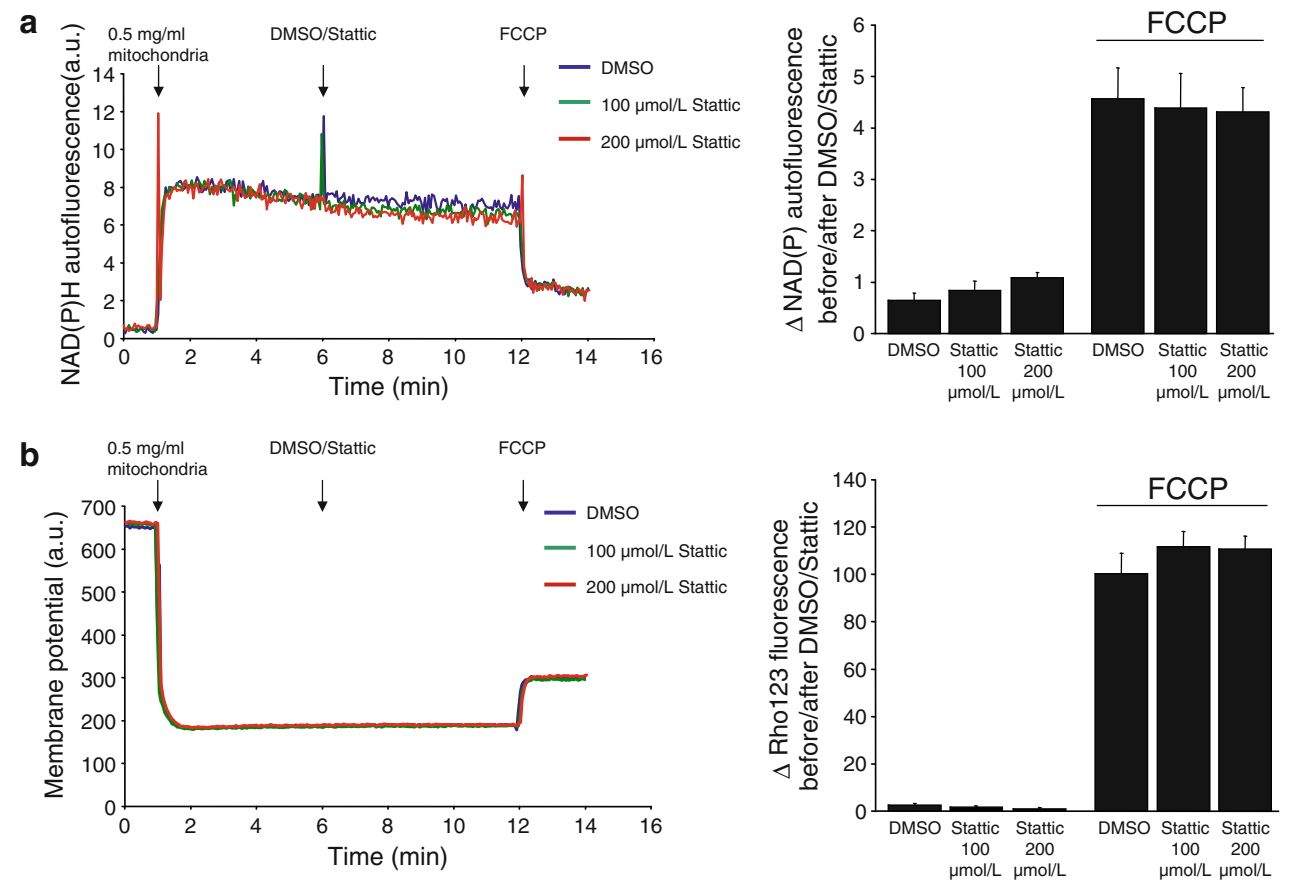

Fig. 12 Stattic causes no acute toxic effects on mitochondria. a $\mathrm{NAD}(\mathrm{P}) \mathrm{H}$ autofluorescence of $0.5 \mathrm{mg} / \mathrm{mL}$ rat left ventricular (LV) subsarcolemmal mitochondria (SSM) was measured before and after the addition of 100 or $200 \mu \mathrm{mol} / \mathrm{L}$ Stattic or DMSO as vehicle. To induce collapse of the mitochondrial membrane potential, $100 \mathrm{nmol} / \mathrm{L}$ FCCP was given. The difference between the NAD(P)H autofluorescence $1 \mathrm{~min}$ before and $1 \mathrm{~min}$ after the addition of Stattic,

(LSM Pascal 5, Zeiss, Jena, Germany) at 630× magnification. For negative control, primary antibodies were omitted. Unless otherwise stated, about 20-30 mitochondria were analyzed from each individual experiment (see Figs. 9, 10, 11, 12).

\section{References}

1. Argaud L, Gateau-Roesch O, Raisky O, Loufouat J, Robert D, Ovize M (2005) Postconditioning inhibits mitochondrial permeability transition. Circulation 111:194-197

2. Boengler K, Buechert A, Heinen Y, Roeskes C, Hilfiker-Kleiner D, Heusch G, Schulz R (2008) Cardioprotection by ischemic postconditioning is lost in aged and STAT3-deficient mice. Circ Res 102:131-135

3. Boengler K, Dodoni G, Rodriguez-Sinovas A, Cabestrero A, Ruiz-Maena M, Gres P, Konietzka I, Lopez-Iglesias C, García-Dorado D, Heusch G, Schulz R (2005) Connexin 43 in cardiomyocyte mitochondria and its increase by ischemic preconditioning. Cardiovasc Res 67:234-244

4. Boengler K, Hilfiker-Kleiner D, Drexler H, Heusch G, Schulz R (2008) The myocardial JAK/STAT pathway: from protection to failure. Pharmacol Therap 120:172-185

5. Boengler K, Konietzka I, Buechert A, Heinen Y, Garcia-Dorado D, Heusch G, Schulz R (2007) Loss of ischemic preconditioning's cardioprotection in aged mouse hearts is associated with
DMSO or FCCP, respectively, was calculated $(n=5)$. b Mitochondrial membrane potential of $0.5 \mathrm{mg} / \mathrm{mL}$ rat LV SSM was measured with rhodamine 123 before and after the addition of 100 or $200 \mu \mathrm{mol} /$ L Stattic or DMSO as vehicle. The difference between the fluorescence 1 min before and 1 min after the addition of Stattic, DMSO or FCCP, respectively, was calculated $(n=5)$

reduced gap junctional and mitochondrial levels of connexin 43 . Am J Physiol Heart Circ Physiol 292:H1764-H1769

6. Boengler K, Schulz R, Heusch G (2009) Loss of cardioprotection with ageing. Cardiovasc Res 83:247-261

7. Boengler K, Stahlhofen S, van de Sand A, Gres P, Ruiz-Meana M, Garcia-Dorado D, Heusch G, Schulz R (2009) Presence of connexin 43 in subsarcolemmal but not in interfibrillar cardiomyocyte mitochondria. Basic Res Cardiol 104:141-147

8. Cohen MV, Yang XM, Downey JM (2008) Acidosis, oxygen, and interference with mitochondrial permeability transition pore formation in the early minutes of reperfusion are critical to postconditioning's success. Basic Res Cardiol 103:464-471

9. Di Lisa F, Bernardi P (2009) A CaPful of mechanisms regulating the mitochondrial permeability transition. J Mol Cell Cardiol 46:775-780

10. Fuglesteg BN, Suleman N, Tiron C, Kanhema T, Lacerda L, Andreasen TV, Sack MN, Jonassen AK, Mjos OD, Opie LH, Lecour S (2008) Signal transducer and activator of transcription 3 is involved in the cardioprotective signalling pathway activated by insulin therapy at reperfusion. Basic Res Cardiol 103:444-453

11. Goodman MD, Koch SE, Fuller-Bicer GA, Butler KL (2008) Regulating RISK: a role for JAK-STAT signaling in postconditioning? Am J Physiol Heart Circ Physiol 295:H1649-H1656

12. Gough DJ, Corlett A, Schlessinger K, Wegrzyn J, Larner AC, Levy DE (2009) Mitochondrial STAT3 supports Ras-dependent oncogenic transformation. Science 324:1713-1716

13. Gross ER, Hsu AK, Gross GJ (2007) Diabetes abolishes morphine-induced cardioprotection via multiple pathways upstream of glycogen synthase kinase-3 beta. Diabetes 56:127-136 
14. Hattori R, Maulik N, Otani H, Zhu L, Cordis G, Engelman RM, Siddiqui MAQ, Das DK (2001) Role of STAT3 in ischemic preconditioning. J Mol Cell Cardiol 33:1929-1936

15. Hausenloy DJ, Maddock HL, Baxter GF, Yellon DM (2002) Inhibiting mitochondrial permeability transition pore opening: a new paradigm for myocardial preconditioning? Cardiovasc Res 55:534-543

16. Hausenloy DJ, Yellon DM, Mani-Babu S, Duchen MR (2004) Preconditioning protects by inhibiting the mitochondrial permeability transition. Am J Physiol Heart Circ Physiol 287:H841H849

17. Heusch G (2001) Nitroglycerin and delayed preconditioning in humans. Yet another new mechanism for an old drug? Circulation 103:2876-2878

18. Heusch G, Boengler K, Schulz R (2008) Cardioprotection: nitric oxide, protein kinases, and mitochondria. Circulation 118:19151919

19. Heusch G, Boengler K, Schulz R (2010) Inhibition of mitochondrial permeability transition pore opening: the holy grail of cardioprotection. Basic Res Cardiol 105:151-154

20. Hilfiker-Kleiner D, Hilfiker A, Fuchs M, Kaminski K, Schaefer A, Schieffer B, Hillmer A, Schmiedl A, Ding Z, Podewski E, Poli V, Schneider MD, Schulz R, Park J-K, Wollert KC, Drexler H (2004) Signal transducer and activator of transcription 3 is required for myocardial capillary growth, control of interstitial matrix deposition, and heart protection from ischemic injury. Circ Res 95:187-195

21. Javadov SA, Clarke S, Das M, Griffiths EJ, Lim KH, Halestrap AP (2003) Ischaemic preconditioning inhibits opening of mitochondrial permeability transition pores in the reperfused rat heart. J Physiol 549:513-524

22. Lacerda L, Somers S, Opie HL, Lecour S (2009) Ischemic postconditioning protects against reperfusion injury via the SAFE pathway. Cardiovasc Res 84:201-208

23. Lecour S (2009) Activation of the protective survivor activating factore enhancement (SAFE) pathway against reperfusion injury: Does it go beyond the RISK path? J Mol Cell Cardiol 47:32-40

24. Lecour S, Suleman N, Deuchar GA, Somers S, Lacerda L, Huisamen B, Opie LH (2005) Pharmacological preconditioning with tumor necrosis factor-a activates signal transducer and activator of transcription-3 at reperfusion without involving classic prosurvival kinases (Akt and extracellular signal-regulated kinase). Circulation 112:3911-3918

25. Levy DE, Lee CK (2002) What does Stat3 do? J Clin Invest 109:1143-1148

26. Matsuyama N, Leavens JE, McKinnon D, Gaudette GR, Aksehirli TO, Krukenkamp IB (2000) Ischemic but not pharmacological preconditioning requires protein synthesis. Circulation 102:312-318

27. Myers MG (2009) Moonlighting in mitochondria. Science 323:723-724

28. Ovize M, Baxter GF, Di Lisa F, Ferdinandy P, Garcia-Dorado D, Hausenloy DJ, Heusch G, Vinten-Johansen J, Yellon DM, Schulz $\mathrm{R}$ (2010) Postconditioning and protection from reperfusion injury: where do we stand? Cardiovasc Res 87:406-423

29. Palmer JW, Tandler B, Hoppel CL (1977) Biochemical properties of subsarcolemmal and inter-fibrillar mitochondria isolated from rat cardiac-muscle. J Biol Chem 252:8731-8739

30. Pfeffer LM, Mullersman JE, Pfeffer SR, Murti A, Shi W, Yang CH (1997) STAT3 as an adapter to couple phosphatidylinositol 3-kinase to the IFNAR1 chain of the type I interferon receptor. S 276:1418-1420

31. Phillips D, Reilley MJ, Aponte AM, Wang G, Boja E, Gucek M, Balaban RS (2010) Stoichiometry of STAT3 and mitochondrial proteins: implications for the regulation of oxidative phosphorylation by protein-protein interactions. J Biol Chem 285:23532-23536

32. Piot C, Croisille P, Staat P, Thibault H, Rioufol G, Mewton N, Elbelghiti R, Cung TT, Bonnefoy E, Angoulvant D, Macia C, Raczka F, Sportouch C, Gahide G, Finet G, Andre-Fouet X, Revel D, Kirkorian G, Monassier J-P, Derumeaux G, Ovize M (2008) Effect of cyclosporine on reperfusion injury in acute myocardial infarction. N Engl J Med 359:473-481

33. Podewski EK, Hilfiker-Kleiner D, Hilfiker A, Morawietz H, Lichtenberg A, Wollert KC, Drexler H (2003) Alterations in janus kinase (JAK)-signal transducers and activators of transcription (STAT) signaling in patients with end-stage dilated cardiomyopathy. Circulation 107:798-802

34. Rasola A, Bernardi P (2007) The mitochondrial permeability transition pore and its involvement in cell death and in disease pathogenesis. Apoptosis 12:815-833

35. Riva A, Tandler B, Loffredo F, Vazquez E, Hoppel C (2005) Structural differences in two biochemically defined populations of cardiac mitochondria. Am J Physiol Heart Circ Physiol 289:H868-H872

36. Rodriguez-Sinovas A, Boengler K, Cabestrero A, Gres P, Morente M, Ruiz-Meana M, Konietzka I, Miró E, Totzeck A, Heusch G, Schulz R, Garcia-Dorado D (2006) Translocation of connexin 43 to the inner mitochondrial membrane of cardiomyocytes through the heat shock protein 90-dependent TOM pathway and its importance for cardioprotection. Circ Res 99:93-101

37. Rottlaender D, Boengler K, Wolny M, Michels G, Endres-Becker J, Motloch LJ, Schwaiger A, Buechert A, Schulz R, Heusch G, Hoppe UC (2010) Connexin 43 acts as a cytoprotective mediator of signal transduction by stimulating mitochondrial KATP channels in mouse cardiomyocytes. J Clin Invest 120:1441-1453

38. Rowland RT, Meng X, Cleveland JC Jr, Meldrum DR, Harken AH, Brown JM (1997) Cardioadaptation induced by cyclic ischemic preconditioning is mediated by translational regulation of de Novo protein synthesis. J Surg Res 71:155-160

39. Skyschally A, Schulz R, Heusch G (2008) Pathophysiology of myocardial infarction: protection by ischemic pre- and postconditioning. Herz 33:88-100

40. Skyschally A, Schulz R, Heusch G (2010) Cyclosporine A at reperfusion reduces infarct size in pigs. Cardiovasc Drugs Ther 24:85-87

41. Smith RM, Suleman N, Lacerda L, Opie LH, Akira S, Chien KR, Sack MN (2004) Genetic depletion of cardiac myocyte STAT-3 abolishes classical preconditioning. Cardiovasc Res 63:611-616

42. Staat P, Rioufol G, Piot C, Cottin Y, Cung TT, L'Huillier I, Aupetit J-F, Bonnefoy E, Finet G, Andre-Fouet X, Ovize M (2005) Postconditioning the human heart. Ciculation 112:21432148

43. Suleman N, Somers S, Smith R, Opie LH, Lecour SC (2008) Dual activation of STAT- 3 and Akt is required during the trigger phase of ischaemic preconditioning. Cardiovasc Res 79:127-133

44. Wegrzyn J, Potla R, Chwae YJ, Sepuri NB, Zhang Q, Koeck T, Derecka M, Szczepanek K, Szelag M, Gornicka A, Moh A, Moghaddas S, Chen Q, Bobbili S, Cichy J, Dulak J, Baker DP, Wolfman A, Stuehr D, Hassan MO, Fu XY, Avadhani N, Drake JI, Fawcett P, Lesnefsky EJ, Larner AC (2009) Function of mitochondrial Stat3 in cellular respiration. Science 323:793-797

45. Xuan Y-T, Guo Y, Han H, Zhu Y, Bolli R (2001) An essential role of the JAK-STAT pathway in ischemic preconditioning. Proc Natl Acad Sci USA 98:9050-9055

46. Yamaura G, Turoczi T, Yamamoto F, Siddqui MA, Maulik N, Das DK (2003) STAT signaling in ischemic heart: a role of STAT5A in ischemic preconditioning. Am J Physiol Heart Circ Physiol 285:H476-H482 